\title{
AUTO-HEMOTERAPIA, INTERVENÇÃO DO ESTADO E BIOÉTICA
}

\author{
Denise Ferreira leite, Patrícia Fernanda Toledo Barbosa, Volnei Garrafa* \\ Trabalho realizado na Cátedra Unesco de Bioética da Universidade de Brasília e na Agência Nacional de Vigilância Sanitária do Ministério da \\ Saúde - Anvisa, Brasília, DF
}

*Correspondência:

Caixa postal 04451

Brasília - DF

Cep 70904-970

bioetica@unb.br

\begin{abstract}
RESUMO
A auto-hemoterapia é uma prática de uso clínico crescente, mas com potencial risco à saúde dos indivíduos, uma vez que se trata de procedimento terapêutico sem comprovação científica. Até o momento não existem estudos clínicos que comprovem a eficácia e a segurança deste procedimento; apenas pesquisas experimentais com resultados questionáveis, tanto em seres humanos quanto em animais. Nos últimos anos, a área de Vigilância Sanitária (VS) do Ministério da Saúde ampliou suas ações preventivas e de controle de riscos tanto no âmbito privado como coletivo. As ações da VS têm, muitas vezes, como base o poder legal de polícia administrativa que a legislação lhe confere. Esse poder é entendido como a faculdade que dispõe a Administração Pública para condicionar e restringir o uso e gozo de bens, atividades e direitos individuais, em benefício da coletividade ou do próprio Estado. Recentemente, o Estado, por meio do poder de polícia da VS, interveio na prática da auto-hemoterapia no Brasil. 0 presente estudo analisa e defende a ação interventiva da VS na prática clínica da auto-hemoterapia no país, tendo como base de sustentação argumentativa os "Q uatro Pês" desenvolvidos pela chamada "Bioética de Intervenção" - prevenção, proteçãa, precauçãa e prudência.
\end{abstract}

Un ITERm os: Auto-hemoterapia. Vigilância Sanitária. Poder de polícia. Bioética de Intervenção. Prevenção, proteção, precaução e prudência.

\section{InTRODUÇÃO}

A defesa e a proteção da saúde surgem no aparato legal brasileiro por meio das constituições federais de 1937, 1946, 1967, culminando com a de 1988. Referem-se à competência do Estado de legislar sobre a saúde, ganhando ênfase com a criação do M inistério da Saúde (1950) e a necessidade de um espaço institucional que acompanhasse 0 desenvolvimento industrial e tecnológico ${ }^{1,2}$. N esse contexto, a Vigilância Sanitária surge inicialmente como um conjunto de ações que visava ao controle dos riscos relacionados à circulação de mercadorias e à força de trabalho ${ }^{3}$. Atualmente, Vigilância Sanitária é entendida como um conjunto de ações capaz de eliminar, diminuir ou prevenir riscos à saúde e de intervir nos problemas sanitários decorrentes do meio ambiente, da produção e circulação de bens e da prestação de serviços de interesse da saúde 4 .

As práticas da Vigilância Sanitária, no entanto, sobretudo com a nova concepção da saúde pública revelada ao final do século XX, ampliam as opções de prevenção e controle de risco no sentido da precaução, implicando não apenas no âmbito individual, mas também na perspectiva coletiva: "0 fenômeno social traduzido no princípio de precaução levou, igualmente, ao desenvolvimento de uma filosofia da precaução, construída com base em uma história da prudência, que revela, a princípio, o domínio do paradigma da responsabilidade, substituído - na passagem para o século XX - pelo da solidariedade. É a segurança - o novo paradigma, em fase de formação - que dá às obrigações morais a forma de ética e transforma o princípio de responsabilidade em precaução" 5 .
Algumas ações da Vigilância Sanitária partem da prerrogativa legal do poder de polícia administrativa. Ao executar tais ações, no entanto, deve-se procurar levar em consideração alguns referenciais éticos que proporcionem sustentação às mesmas como os "Q uatro Pês" - prevenção, proteção, precaução e prudência - desenvolvidos pela chamada Bioética de Intervenção ${ }^{6} .0$ poder acima referido é entendido como a faculdade que dispõe a Administração Pública para condicionar e restringir 0 uso e gozo de bens, atividades e direitos individuais, em benefício da coletividade ou do próprio Estado ${ }^{7}$. Assim, sua finalidade é a proteção do interesse público no seu sentido mais amplo, sendo seus limites demarcados pela conciliação entre o interesse social com os direitos fundamentais do indivíduo.

Cabe ressaltar que esse poder de agir da autoridade pública converte-se em dever de agir, uma vez que não se admite omissão diante de situações em que se exige sua atuação. A atuação do poder de polícia se dá por meio de ordens, proibições, sançôes, normas limitadoras e sancionadoras de conduta na utilização de bens ou no exercício de atividades sujeitas ao policiamento administrativo ${ }^{8}$.

A auto-hemoterapia é uma prática de freqüência crescente, com potencial risco à saúde dos indivíduos, pois é um método terapêutico sem comprovação científica, executado muitas vezes por pessoal sem capacitação e sob condições inadequadas de Biossegurança. Praticamente, não há estudos clínicos que comprovem a eficácia e a segurança deste procedimento; apenas pesquisas experimentais com resultados questionáveis, tanto em estudos com seres humanos quanto em animais. 
Considerando a legitimidade da intervenção do Estado frente a situações de interesse coletivo, vulnerabilidade e suscetibilidade, 0 presente estudo se propõe a analisar a prática da auto-hemoterapia frente às ações de Vigilância Sanitária e sua relação com os "Q uatro Pês" da Bioética de Intervenção.

\section{Apresentação do problema}

\section{A auto-hemoterapia}

A auto-hemoterapia consiste na retirada de sangue por punção venosa e sua imediata administração por via intramuscular ou subcutânea, em que o doador e o receptor são o mesmo indivíduo. Também é conhecida como terapia do soro, imunoterapia ou autohemotransfusão $0^{\text {9,10,11. }}$.

Consta na escassa literatura existente sobre 0 assunto que a autohemoterapia foi introduzida como tentativa terapêutica por Ravaut, por volta de 1910 e, desde então, tem sido utilizada como tentativa de tratamento de diversos problemas de saúde, tanto em humanos quanto em animais ${ }^{10,12}$. Apóia-se na comparação do procedimento à aplicação de uma vacina autógena, estimulando a resposta imune do organismo diante de uma série de problemas, infecciosos ou não, cuja explicação se baseia no raciocínio do foco de infecção ${ }^{12,13,14}$.

No entanto, quando se buscam referências sobre o tema, os artigos encontrados, além de não-indexados na sua grande maioria, referemse a relatos de experiências e de casos sem condução metodológica que apontem grau de relevância científica, a ponto de indicar o procedimento na terapia de pacientes. Resultados de estudos conduzidos de forma mais criteriosa em bovinos não apontam diferença estatística entre a recuperação de animais tratados com protocolos experimentais contendo ou não a auto-hemoterapia ${ }^{12}$.

A despeito das escassas e controversas evidências científicas, há médicos, enfermeiros, auxiliares de enfermagem e pessoas sem habilitação comercializando o procedimento nos meios de comunicação. Além disso, a prática pode causar reações adversas imediatas ou tardias de gravidade imprevisível no paciente, aumentando o risco e a gravidade destas reações quando realizada por pessoas não habilitadas ou pelo próprio paciente.

A Agência Nacional de Vigilância Sanitária (Anvisa) e a Sociedade Brasileira de Hematologia e Hemoterapia (SBHH), por meio da Nota Técnica $n-1 / 2007^{15}$ e do Comunicado Eletrônico AutoHemoterapia ${ }^{16}$, respectivamente, não reconhecem a autohemoterapia como prática hemoterápica. Em sua nota técnica, a Anvisa orientou as vigilâncias sanitárias estaduais e municipais a enquadrar 0 procedimento como infração sanitária, segundo o Decreto no. 77.0521 $1976^{17}$, considerando sua prática sujeita às penalidades previstas pela Lei $n^{0} 6.437 / 1977^{18}$.

0 Conselho Federal de Medicina é ainda mais contundente ao emitir oficialmente o parecer $n-12 / 2007^{19}$ sobre a auto-hemoterapia, ao manifestar que a mesma "não foi submetida a testes genuínos, não foi corroborada, e nada há, além de indícios, casos isolados, narrados com dramaticidade, que pouco se prestam a provar coisa alguma perante a ciência em que ampare o seu valor, sendo seu uso atual em seres humanos uma aventura irresponsável".

\section{0 poder de polícia}

A Lei no 8080/1990, ou Lei 0 rgânica da Saúde4, atribui à U nião, aos Estados, aos municípios e ao Distrito Federal o exercício do poder de polícia sanitária. Assim, a descentralização político-administrativa faz com que o poder de polícia possua competências exclusivas e concorrentes nas três esferas estatais. Com a Lei n- 9782/199920, à Anvisa foi atribuída a finalidade de: "promover a proteção da saúde da população, por intermédio do controle sanitário da produção e da comercialização de produtos e serviços submetidos à Vigilância Sanitária, inclusive dos ambientes, dos insumos e das tecnologias a eles relacionados, bem como o controle de portos, aeroportos e fronteiras".

$\mathrm{N}$ a sua origem, o poder de polícia era exercido por pessoas ou instituições com ações legitimadas pela sociedade para exercer a vigilância pública, penalizando os indivíduos que não cumprissem a ordem social estabelecida. A necessidade de proteção dos habitantes da polis, gerou o termo politia e o vernáculo polícia ${ }^{7,21}$. Com 0 ordenamento jurídico do Estado Democrático de Direito, esse poder passou a ter limitações e imposições legais para o seu exercício, sendo considerado um ato administrativo.

Atualmente, a função do poder de polícia administrativa é garantir a supremacia dos direitos coletivos sob os direitos individuais quando há oposição entre ambos, tendo como objetivo bens, direitos e atividades individuais ${ }^{22}$. Seus atributos são os seguintes: a discricionariedade no uso da liberdade legal de valoração das atividades policiadas e na graduação das sanções; a auto-executoriedade do ato independente de mandato judicial, excetuando-se as multas e demais prestações pecuniárias; e a coercibilidade do ato pela imposição coativa das medidas adotadas, admitindo até o uso da força pública nos casos de resistência por parte do administrado $7,21,22$.

Por impor limites administrativos à liberdade e à propriedade, o poder de polícia choca-se com os princípios relacionados aos direitos individuais, principalmente com a autonomia, princípio familiar ao campo da bioética. Interpretações errôneas de termos, conceitos e referenciais pertencentes às duas áreas relacionadas à questão - Vigilância Sanitária e Bioética - têm tornado o assunto palco de algumas contradições. A Bioética oferece ferramentas e subsídios para auxiliar à Vigilância nos momentos em que tais contradições entre referenciais bioéticos e sanitários aparecem. Segundo Arreguy e Schramm ${ }^{23}$, a Bioética foi criada na tentativa de tentar compreender e dissolver conflitos de interesses e valores no campo da saúde, sendo uma ferramenta no auxílio da prática gestora de serviços públicos de saúde.

\section{Referencial teórico - Bioética de intervenção e os "Q uatro Pês" para uma prática ética responsável: prevenção, prote- ção, precaução e prudência}

A Bioética de intervenção considera os "Q uatro Pês" - prevenção, proteção, precaução e prudência - como referenciais teóricos e práticos indispensáveis em questões que envolvam o uso de tecnologias em situações de vulnerabilidade, gestão da "coisa pública" e equilíbrio ambienta ${ }^{24}$. 0 tema da prevenção é usado em questões que envolvam possiviveis danos e iatrogenias de tecnologias existentes; a precaução é evocada em situações em que se desconhecem os riscos envolvidos; a prudência é lembrada com relação aos cuidados necessários frente aos 
avanços tecnológicos; enquanto o referencial da proteção objetiva trabalhar o tema da vulnerabilidade, da proteção indispensável aos mais frágeis, aos necessitados ${ }^{6,24}$.

0 conceito de risco é importante na compreensão dos "Q uatro Pês". Risco é a chance ou possibilidade de ocorrência de uma consequiência prejudicial ou ruim em virtude de uma ação ou omissão. Refere-se à possibilidade, com certo grau de probabilidade, de dano à saúde, ambiente ou aos produtos em combinação com a natureza e magnitude do dano². Faz-se necessário explicitar, também, 0 conceito de vulnerabilidade, diferenciando-o do conceito de risco. Para Sánchez et al. ${ }^{25}$, a vulnerabilidade é como um conjunto de aspectos que ultrapassam 0 individual, abrangendo aspectos coletivos e contextuais que levam à suscetibilidade a doenças ou agravos, além de aspectos relacionados à disponibilidade ou à carência de recursos destinados à proteção.

\section{Prevenção}

A idéia de prevenção pode ser trazida da medicina preventiva, referindo-se à situação em que condutas são tomadas com o objetivo imediato de que não ocorra o processo saúde- doença - ou, no caso do uso de tecnologias, o dano - e, para isto, busca-se interferir nos fatores predisponentes, na educação e na mudança de hábitos, dentre outros ${ }^{1,23}$. 0 dano é tido como uma ameaça ou um prejuízo que seguramente ocorrerá, e, dentro deste contexto, o risco é a possibilidade de dano. Para Engelhardt, as "políticas para enfrentar a ameaça de risco levantam a questão da comparação entre danos e benefícios atuais com possíveis danos e benefícios futuros" 26 .

A prevenção, assim, antecipa-se às possibilidades de danos à saúde sendo o referencial que busca a ação antecipada e, para isso, "é necessário ter conhecimentos e certezas científicas dos efeitos dos atos, processos ou produtos" ${ }^{27}$. Berlinguer ressalta que, além da "virtude antecipatória", a prevenção também tem como motivações éticas: um caráter igualitário, pois quando praticada, elimina a desigualdade entre uma pessoa acometida por uma doença e uma pessoa que permanece com boa saúde; e ainda, a atenuação de conflitos entre interesses e mesmo entre valores humanos dificilmente conciliáveis entre si27.

Czeresnia define prevenção como uma intervenção direta para evitar a emergência de doenças específicas, visando reduzir a incidência e prevalência das mesmas na população. Dessa forma, a prevenção se baseia no conhecimento epidemiológico. Esta autora diferencia a prevenção da promoção, considerando a última mais ampla que a primeira. Para ela, a promoção estimula a capacidade individual e coletiva de escolha, com base no conhecimento e atribuindo valores, ou seja, estimulando a autonomia ${ }^{28}$.

\section{Proteção}

0 referencial da proteção é entendido como uma especificação do princípio da responsabilidade para Schramm e Kottow ${ }^{23,29}$, uma vez que é aplicado em situações de fragilidade e ameaça à população, ou seja, em situações de vulnerabilidade e suscetibilidade. Kottow diferencia vulnerabilidade de suscetibilidade, sendo a primeira "... atributo antropológico de todo ser humano" e a segunda, "... um dano instalado em grupos sociais e indivíduos" ${ }^{30}$. Assim, 0 objetivo da proteção é normatizar as práticas humanas por meio da antecipação dos efeitos positivos, prevenindo eventuais efeitos negativos.
A proteção seria a ferramenta principal para avaliar moralmente as políticas públicas em saúde, visando justiça social. Dessa forma, 0 referencial da proteção deve ser considerado nas seguintes circunstâncias: existência de objetivos sanitários inevitáveis e indispensáveis; existência de medidas necessárias e razoáveis com alta probabilidade de prevenção dos problemas sanitários por meio de programas de saúde; e existência de necessidade social para o exercício da proteção, o que justifica a não interferência dos efeitos negativos na validação do programa ${ }^{31}$.

Pontes et al. ${ }^{32}$ definem proteção como o resguardo ou cobertura das necessidades essenciais, garantindo 0 atendimento de requerimentos moralmente legítimos de todos os indivíduos. Para estes autores são consideradas necessidades essenciais: a saúde, a educação, a alimentação, a segurança, a moradia, 0 vestuário e 0 abastecimento de água. A partir do século XVIII, o Estado passou a resguardar os interesses individuais dos cidadãos, caracterizando como primeiro nível de proteção os direitos humanos fundamentais da pessoa, também conhecidos como direitos liberais. 0 segundo nível de proteção refere-se à população, com atuação no campo dos direitos sociais.

\section{Precaução}

0 desenvolvimento da ciência tem produzido avanços tecnológicos em diversas áreas do conhecimento. $\mathrm{N}$ a área da saúde, riscos potenciais e reais foram criados por este avanço tecnológico. Assim, o referencial da precaução busca a avaliação segura e quantitativa dos riscos por meio da eliminação ou diminuição do dano causado pelos mesmos; atuando tanto na possibilidade como no próprio dano. Existem muitos conceitos e definições para precaução em instrumentos internacionais, aparecendo pela primeira vez no sistema das N ações Unidas, na Declaração do Rio sobre Ambiente e Desenvolvimento, em $1992{ }^{33}$.

A precaução nasceu em considerações sobre meio ambiente, com a noção de controle pré-dano dos riscos (medidas de antecipação) e responsabilidade ética. Assim, a sua finalidade é a proteção dos seres humanos e do ambiente contra possíveis riscos da ação humana por meio de medidas de controle pré-dano. A aplicação deste referencial ocorre nas seguintes situações ${ }^{33}$ : existência de incertezas científicas sobre a causalidade, magnitude, probabilidade e natureza do dano; existência de hipóteses ou modelos, baseados em evidências científicas, do possível dano; redução impossivel das incertezas sem um aumento na ignorância de outros fatores relevantes; dano potencial sério, irreversível ou moralmente inaceitável à geração atual ou futura; e necessidade de ação imediata, uma vez que tardiamente seria mais difícil ou com mais custo.

A Organização das Nações Unidas para a Educação, a Ciência e a Cultura (UN ESCO ) relaciona à precaução a ignorância culposa, a coresponsabilidade e a justiça intra-geração nas ações ou omissões de indivíduos, instituições e Estados. 0 s dois primeiros, ignorância culposa e co-responsabilidade, dizem respeito à responsabilidade, não isentando a mesma por falta de conhecimento ou exercício profissional. A justiça intrageração é a igual distribuição de oportunidades para cada indivíduo em ter segurança econômica, social e política. A UN ESCO diferencia os termos precautionary principle e precautionary approach, sendo o primeiro relacionado à base filosófica da precaução, enquanto o segundo é a aplicação prática da precaução ${ }^{33}$. 
Soule divide a regulação pela precaução em duas categorias: weak precautionary e strong precautionary, sendo a diferença entre elas a necessidade de dispositivos de ação regulatória na última. No caso da weak precautionary, um risco poderia ser aceito em virtude de um benefício ou por ser muito caro evitá-lo, necessitando seu gerenciamento. Já na regulação por strong precautionary, o risco é evitado ${ }^{34}$.

Segundo Dallari et al. ${ }^{5}$, a precaução "pretende conter a inovação, re-orientando o progresso científico ilimitado e re-valorizando a busca dos verdadeiros responsáveis pelos comportamentos imprudentes". Para estas autoras, 0 "agir em saúde pública significa conduzir uma ação política, que, por sua vez, obriga à prudência", despertando os Estados para a proteção e prevenção da saúde pública. "De maneira geral, 0 escopo da precaução é ultrapassar a prevenção. N ão seria mais preciso que um dano se produzisse, ou se mostrasse iminente, para que um gesto, visando evitar a produção ou a repetição desse dano, fosse legítimo." Para Weed, a precaução tem como idéia central a ação preventiva antecipatória devido à evidência científica de incerteza frente a um risco, estando a prevenção dentro da precaução ${ }^{35}$.

\section{Prudência}

A prudência é uma das quatro virtudes cardeais da Antiguidade e da Idade Média, sendo as demais, a justiça, a coragem e a temperança. Atualmente, a prudência teve seu significado reduzido ao aspecto de cuidado, cautela, risco, bem diferente do significado dado por Aristóteles e Tomás de Aquino. Aristóteles considerava a prudência uma virtude, a moderação, o ponto de equilíbrio entre os dois extremos de vício. A prudência era vista como necessária para as demais virtudes por ser uma qualidade que determinaria a conduta do homem por meio da razão e da verdade ${ }^{36}$. Para ele, o homem prudente delibera e julga as coisas de modo conveniente, buscando a felicidade e uma vida boa. Contudo, Aristóteles não considerava a prudência pertencente à arte ou à ciência. Além disso, Aristóteles diferencia a prudência da sabedoria, sendo a primeira a sabedoria prática (phrósnesis) e a segunda a sabedoria teórica (sophia).

Tomás de Aquino ${ }^{37}$ define prudência como "a virtude da decisão certa" ou "recta ratio agibilium" (reta razão aplicada ao agir). Para ele, a prudência regeria as demais virtudes cardeais, não sendo nem arte nem ciência. Ele acreditava que a prudência era uma virtude composta por partes, existindo três tipos: partes integrantes, partes subjetivas e partes potenciais. As partes integrantes seriam as funções da virtude, ocorrendo para 0 ato perfeito da virtude da prudência enquanto cognoscitiva (memória, razão, intelecto, docilidade, sagacidade) e preceptiva (previdência, circunspeção, precaução), esta última aplicando o conhecimento à ação. Com relação à previdência, Tomás de Aquino a define como um termo que "... implica, com efeito, que o olhar se prenda a qualquer coisa distante como a um termo ao qual devem ser ordenadas as ações presentes". Para ele, a precaução é necessária à prudência "... para escolher os bens e evitar os males" 37 .

As partes subjetivas compõem a variabilidade de prudência existente no mundo para o "governo da multidão", enquanto as potenciais dizem respeito aos atos secundários relacionados ao ato da prudência. Compõe a parte subjetiva da prudência o componente legislativo, político, econômico e militar. A eubulia, ou ato de bem deliberar ou 0 que aconselha bem, integra a parte potencial, assim comosynesis, juízo reto de ações particulares, e a gnome, esta implicando em uma certa perspicácia no julgamento. Um dos vícios opostos à prudência é a imprudência, definida como a falta da primeira. Fazem parte da imprudência a negligência (falta de solicitude devida), precipitação ou temeridade (falta de deliberação, esta ocupação da eubulia), inconsideração (falta de julgamento, objeto dasynesis e gnome) e a inconstância (abandono de um bom propósito determinado) ${ }^{37}$.

Gracián ${ }^{38}$ é outro filósofo que trata do princípio da prudência. Para ele, a prudência é composta, dentre outros atributos, pelo conhecimento, discernimento, sabedoria, inteligência, razão, reflexão, ponderação, percepção, decisão, benevolência, beneficência, condescendência e sensatez.

\section{Discussão}

A Bioética de intervenção considera como moralmente justificável no campo público e coletivo, dentre outros aspectos, a priorização de políticas e ações que privilegiem um maior número de pessoas, pelo maior espaço de tempo e que resultem nas melhores consequêencias. No campo privado e individual, defende a busca por soluções viáveis e práticas para os conflitos, contextualizando-os onde os mesmos ocorrem ${ }^{6,24}$

Dentro da Bioética de intervenção, os "Q uatro Pês" são referenciais teóricos e práticos referidos frente a novas tecnologias de serviços e produtos para a saúde, bem como os procedimentos por eles utilizados. A prudência e a precaução são categorias buscadas no momento de desconhecimento dessas novas tecnologias. A proteção e a prevenção, muito conhecidas da Vigilância Sanitária, são referências utilizadas para evitar possíveis danos e iatrogenias advindos do uso dessas tecnologias, uma vez que a população doente que necessita das mesmas é vulnerável e suscetível, ao mesmo tempo, aos possíveis riscos associados.

A Bioética tem muito a contribuir para a Vigilância Sanitária, uma vez que oferece "ferramentas" que poderão ser utilizadas em situações de conflito ético e moral da área da Saúde Pública. Os "Q uatro Pês" são algumas dessas ferramentas, que muito têm a oferecer à temática aqui discutida, principalmente da regulamentação de novas tecnologias. 0 Estado, por meio do poder de polícia da Vigilância Sanitária nos três níveis de governo, interveio na prática da auto-hemoterapia no país, com base nos referenciais bioéticos da prevenção, proteção, precaução e prudência, de forma inter-relacionada e complementar.

Com relação à intervenção do Estado na prática da autohemoterapia, a prudência possui maior abrangência, incluindo a precaução no seu campo de ação. Para evitar os danos oriundos dos riscos inerentes aos serviços e produtos para a saúde, a precaução utiliza a proteção e a prevenção, além de outros conceitos e referenciais. A proteção também utiliza a prevenção na sua ação, se relacionando com as políticas públicas de saúde e sua normatização. A prevenção seria a primeira categoria a ser utilizada na fundamentação da intervenção do Estado devido à relação risco-dano dentro do contexto da saúde pública.

A responsabilidade é outro ponto em comum entre a precaução e a proteção, uma vez que está inserida no discurso de ambas, 
principalmente quando se fala da responsabilidade do Estado na Saúde Pública. C abe resgatar a prudência na atuação do Estado, destacando a sua parte subjetiva como proposto por Tomás de Aquino.

No caso da auto-hemoterapia, a Vigilância Sanitária, por meio do poder de polícia, convoca os "Q uatro Pês" na sua atuação. A prudência $\mathrm{e}$, conseqüentemente, a precaução, relacionam-se ao pouco conhecimento, cientificamente comprovado, desse procedimento. A proteção e a prevenção visam eliminar os danos à saúde da população doente que procura a auto-hemoterapia como tratamento, uma vez que tal procedimento oferece risco de reação adversa, principalmente se realizado em condições sanitárias inadequadas (higiene do local, estrutura física, material descartável, medicamentos e equipamentos de pronto-atendimento, licença ou alvará sanitário do local, recursos humanos capacitados e treinados, etc.).

Q uanto aos recursos humanos, como a prática da autohemoterapia não tem ainda um nível reconhecido de evidência científica, não há pessoas capacitadas e treinadas para sua realização. A falta de conhecimento sobre o procedimento auto-hemoterápico gera desconhecimento sobre as indicações, contra-indicações, posologia, dosagem, interações medicamentosas, reações adversas, entre outras informações necessárias para seu uso terapêutico.

Sem aprofundar especificamente na discussão sobre o emprego dos termos vulnerabilidade e suscetibilidade, a fragilidade do enfermo é um fato concreto: ultrapassa a dimensão biológica, incorporando aspectos psicossociais e afetivos. Essa fragilidade 0 torna consumidor-alvo da promoção de novas tecnologias, nem sempre com resultados terapêuticos comprovados pela ciência, 0 que reforça o poder-dever do Estado de intervir no cuidado ético com a saúde dos seus cidadãos.

\section{Considerações finais e recomendações}

A incorporação às práticas de saúde de tecnologias novas ou decorrentes de conhecimentos tidos como tradicionais em diferentes civilizações, como é o caso da auto-hemoterapia, merece uma racionalização não apenas do ponto de vista de custos, mas visando à segurança de seu uso. Frente às atribuições precípuas da Vigilância Sanitária, os "Q uatro Pês" da Bioética de intervenção e sua prática mostram-se como uma base epistemiológica adequada de argumentação que deve preceder à concessão de permissões e aplicação de penalidades, conferidas pelo poder de polícia a essa função do Estado sobre a Saúde Pública. E, sendo assim, recomenda-se que:

- Profissionais de saúde, além de se manter permanentemente atualizados quanto aos conteúdos técnico-científicos que validem suas práticas e promovam o bem-estar de seus pacientes, estejam atentos às recomendações das autoridades sanitárias e abstenham-se de pressões, mesmo que de origem cultural, para a aplicação de procedimentos sem a devida comprovação científica;

- Conselhos de Classe orientem os profissionais sob sua responsabilidade para essas questões e aumentem a fiscalização sobre o exercício das profissões de saúde, identificando e coibindo as formas indevidas ou deletérias aos pacientes;

- 0 sistema de saúde e a academia estimulem a reflexão e a pesquisa sobre métodos pretensamente terapêuticos, no sentido de se recomendar com segurança o seu uso ou proscrevê-lo, minimizando especulações a respeito e, com isto, conscientizando mais assertivamente a população sobre o seu uso.

\section{Conflito de interesse: não há}

\section{SUMmarY}

Autohemoteraphy, state intervention and bioethics

The increasingly frequent practice of autohemotherapy entails a potential risk to the health of individuals since it is scientifically unproven. There are practically no clinical studies show ing the efficacy and safety of this procedure; only experimental research with questionable results from studies on human beings and animals. 0 ver the last years, the sanitary surveillance area has expanded its preventive and risk control actions based upon a precautionary philosophy in the private as well as the public sphere. By observing the theoretical and practical reference points of the "four Ps" (prevention, protection, precaution and prudence), in accordance with the epistemology developed within Intervention Bioethics, sanitary surveillance actions are based upon legal administrative policing powers. These powers are understood to be the Public Administration's competence to set conditions on and restrict the use and benefit of goods, activities and individual rights, on behalf of the common well being of people or the State itself. The Brazilian State, through its sanitary surveillance policing powers at the three levels of government, has intervened in the practice of autohemotherapy. Considering the responsible State intervention in situations of collective interest, vulnerability and susceptibility, the present study proposed to analyze the practice of autohemotherapy in the light of sanitary surveillance actions and their relationship with the "four Ps" of Intervention Bioethics. [Rev Assoc Med Bras 2008; 54(2): 183-8]

Key wORDS: Autohemotherapy. Policing powers. Sanitary surveillance. Intervention bioethics. Prevention, protection, precaution and prudence.

\section{RefERÊNCIAS}

1. Costa EA. Conceitos e área de abrangência. In: Rozenfeld $S$, editor. Fundamentos da vigilância sanitária. Rio de Janeiro: Editora FIOCRUZ; 2000. p. 41-7.

2. Souto AC. Saúde e política: a vigilância sanitária no Brasil 1976-1994. São Paulo: Sociedade Brasileira de Vigilância Sanitária (SO BRAVIME); 2004. p.227.

3. Costa EA. Vigilância sanitária: proteção e defesa da saúde. $2^{a}$ ed. São Paulo: Sociedade Brasileira de Vigilância de M edicamentos; 2004. p.496.

4. Brasil. Lei №. 8080, de 19 de setembro de 1990. Dispõe sobre as condições para a promoção, proteção e recuperação da saúde, a organização e o funcionamento dos serviços correspondentes. Diário O ficial União, Brasília (DF) 1990; 20 de setembro.

5. Dallari SG, Ventura DFL. O princípio da precaução: dever do Estado ou protecionismo disfarçado? São Paulo Perspect. 2002;16(2):53-63.

6. Garrafa V, Porto D. Intervention bioethics: a proposal for peripheral countries in a context of power and injustice. Bioethics; 2003, 17(56):399-416

7. Meirelles $H L$, Azevedo EA, Aleixo DB, Filho JEB. Direito administrativo brasileiro. 29a ed. São Paulo: M alheiros Editores; 2004. p.798.

8. Dias H P. Direitos e obrigações em saúde. Brasília: Agência Nacional de Vigilância Sanitária (AN VISA); 2002. p.387. 
9. M ettenleiter $M$ W . Autohemotransfusion in preventing postoperative lung complications. Am J Surg. 1936;32(2):321-3.

10. Shakman SH. Autohemoterapia: a picada mágica? [citado 6 jul 2007]. Disponível em: http://paginas.terra.com.br/saude/Autohemoterapia/ AUTOHEM OTHERAPY-_THE_MAGIC-_.SHOT-_Stuart_Shakman.pdf

11. Veronesi R. Imunotera pia:o impacto médico do século. M edicina de $\mathrm{H}$ oje; 1976. [citado 6 jul 2007]. Disponível em: http://paginas.terra.com.br/ saude/Autohemoterapia/IMUNOTERAPIA_Dr_Ricardo_ Veronesi 1976.pdf.

12. Santini API, Britto LAB. Estudo da papilomatose cutânea em bovinos leiteiros: comparação de diferentes tratamentos. CAB. 2004;5(1):39-45.

13. Geovanini T, N orberto M M C. Tratamento de feridas através da autohemoterapia: um estudo de caso clínico [trabalho]. Juiz de Fora: Faculdade de Enfermagem, Universidade Presidente Antônio Carlos, 2007. [citado 6 jul 2007]. Disponível em: http://paginas.terra.com.br/saude/ Autohemoterapia/TRATAM EN TO_DE_FERIDAS_ATRAVES_DA_ Prof_Telma_Giovani.pdf.

14. Silvā M CS. Auto hemoterapia [trabalho de conclusão de curso]. Juiz de Fora: Faculdade de Ciências da Saúde, Universidade Presidente Antônio Carlos; 2006. p.95.

15. Brasil. Agência Nacional de Vigilância Sanitária (Anvisa). N ota Técnica no 1, de 13 de abril de 2007. [citado 6 jul 2007]. Disponível em: http:// www .anvisa.gov.br/divulga/informes/2007/130407.htm.

16. Brasil. Sociedade Brasileira de Hematologia e Hemoterapia (SBHH). Auto-hemoterapia. [citado 6 jul 2007]. Disponível em: http:/l www.sbhh.com.br/home/imunoterapia.htm.

17. Brasil. Decreto №. 77052, de 19 de janeiro de 1976. Dispõe sobre a fiscalização sanitária das condições de exercício de profissões e ocupações técnicas e auxiliares, relacionadas diretamente com a saúde. Diário Oficial União, Brasília (DF) 1976; 20 de janeiro.

18. Brasil. Lei №. 6437, de 20 de agosto de 1977. Configura infrações à legislação sanitária federal, estabelece as sanções respectivas, e dá outras providências. Diário 0 ficial União, Brasília (DF) 1977; 24 de agosto.

19. Brasil. Conselho Federal de Medicina. Parecer no. 12/07. ProcessoConsulta no. 4245/07. Auto-hemoterapia. [citado 6 jul 2007]. Disponível em: http://w w w . portalmedico.org. br/parecer/cfm/2007/12- 2007.htm.

20. Brasil. Lei no. 9782, de 26 de janeiro de 1999. Define 0 Sistema $\mathrm{N}$ acional de Vigilância Sanitária, cria a Agência Nacional de Vigilância Sanitária, e dá outras providências. Diário 0 ficial U nião, Brasília (DF) 1999; 27 janeiro.

21. Cordeiro RGF, M ello DR, M anso M EG, N echio M, Carvalho W. Poder de polícia e as ações de vigilância sanitária. Integração. 2006;12(45):161-9.

22. Barros JW . 0 exercício do poder de polícia na vigilância sanitária do Distrito Federal. In: Brasil. M inistério da Saúde. Fundação 0 swaldo Cruz. Questões atuais de direito sanitário. Brasília: Editora M S; 2006. p.97-120.

23. Arreguy EEM, Schramm FR. Bioética do Sistema Único da Saúde/SU S: uma análise pela bioética da proteção. Rev Bras Cancerol. 2005;51(2):117-23.
24. Garrafa V. Da bioética de princípios a uma bioética interventiva. Bioética. 2005;13(1):125-34.

25. Sánchez AIM, Bertolozzi M R. Pode o conceito de vulnerabilidade apoiar a construção do conhecimento em Saúde Coletiva? [citado 6 jul 2007]. Cienc Saúde Coletiva. 2007;12(2):319-24. Disponível em: http:// w w w . scielosp.org/scielo. php?script = sci_arttext $\&$ pid $=$ S1 413 . $81232007000200007 \& \mathrm{lng}=\mathrm{pt} \& \mathrm{nrm}=$ iso.

26. Engelhardt HT. A bioética do risco: enfrentando a finitude humana. In: Schramm FR, Rego S, Braz M, Palácios M, organizadores. Bioética: riscos e proteção. Rio de Janeiro: Editora UFRJ; 2005. p.133-47.

27. Berlinguer G. Bioética da prevenção [citado 13 nov 2007]. Disponível em: http:w w w . portalmedico.org.br/revista/bio2 v2/bioprev. html.

28. Czeresnia $D$. The concept of health and the difference between prevention and promotion. Cad Saúde Pública. 1999;15(4):701-9.

29. Schramm FR, Kottow M. Princípios bioéticos en salud pública: limitaciones y propuestas. Cad Saúde Pública. 2001;17(5): 949-56.

30. Kottow M. Bioética de proteção: considerações sobre o contexto latinoamericano. In: Schramm FR, Rego S, Braz M, Palácios M, organizadores. Bioética: riscos e proteção. Rio de Janeiro: Editora UFRJ; 2005. p.29-44.

31. Schramm FR. A moralidade da biotecnociência: a Bioética da proteção pode dar conta do impacto real e potencial das biotecnologías sobre a vida e/ou a qualidade de vida das pessoas humanas? In: Schramm FR, Rego $S$, Braz M, Palácios $M$, organizadores. Bioética: riscos e proteção. Rio de Janeiro: Editora UFRJ; 2005. p.15-28.

32. Pontes CAA, Schramm FR. Bioética da proteção e papel do Estado: problemas morais no acesso desigual à água potável. Cad Saúde Pública. 2004;20(5):1319-27.

33. United Nations Educational, Scientific and Cultural 0 rganization. W orld Commission on the Ethics of Scientific Knowledge and Technology (CO M EST). The precautionary principle. Paris: UNESC 0 ; 2005. p.54.

34. Soule $E$. The precautionary principle and the regulation of $U . S$. Food and Drug Safety. J M ed Philos. 2004;29 (3):333-50.

35. W eed DL. Precaution, prevention, and public health ethics. J M ed Philos. 2004; 29(3):313-32.

36. Silva $\mathrm{CH}$. Virtudes e vícios em Aristóteles e Tomás de Aquino: oposição e prudência. Campinas: Boletim do CPA. 1998;5/6:129-40.

37. Aquino T. Suma teológica V - A fé - A esperança - A caridade - A prudência. II Seção da II Parte - Q uestões 1-56. São Paulo: Edições Loyola; 2004. p.682.

38. Gracián B. A arte da prudência. São Paulo: Martin Claret; 2006. p.151.
Artigo recebido: 14/01/08 Aceito para publicação: 14/03/08 